\title{
Improvised Electrsopinning Set up for Thicker Ceramic Nanofiber Mat for High Power Targets
}

Sujit Bidhar ${ }^{1}$, Bob Zwaska ${ }^{1}$, Patrick Hurh ${ }^{1}$

${ }^{1}$ Fermi National Accelerator Lab, Batavia, IL-60510

FERMILAB-POSTER-19-139-AD

\section{Introduction and Objectives}

- In high energy particle physics there is a demand for multiMW high performance particle production targets.

- Nanofiber microstructure will have better performance than current solid targets in mitigating increased thermal tress waves, radiation damage.

- Objective is to fabricate thicker ceramic/metallic nano-fiber with high strength, thermal shock resistance using low cost electrospinning process.

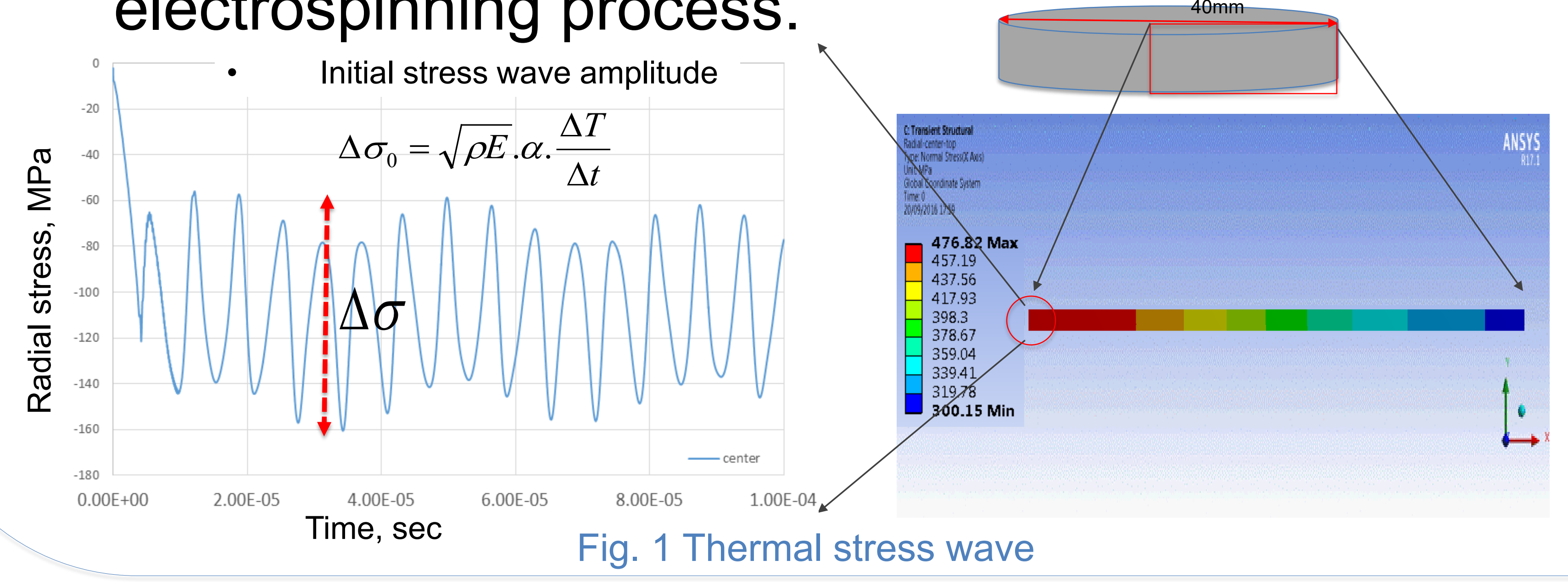

\section{Improvised electrospin set up}



Fig. 3 Lab scale electrospin unit with dual polarity power supply

- Much safe to use $(120 W \rightarrow 4 W !)$

- Mobile compact unit $\rightarrow$ Can be run on 9 or $12 \mathrm{~V}$ battery

- Dual polarity operation

\section{Ceramic/metallic nanofiber production}

Inorganic precursor:

(Zirconium Carbonate +Acetic Acid $\rightarrow$ Zirconia)

$$
\text { 는 }
$$

Polymer solution : PVP+Ethanol/Aceton

Calcination (Heat treatment)
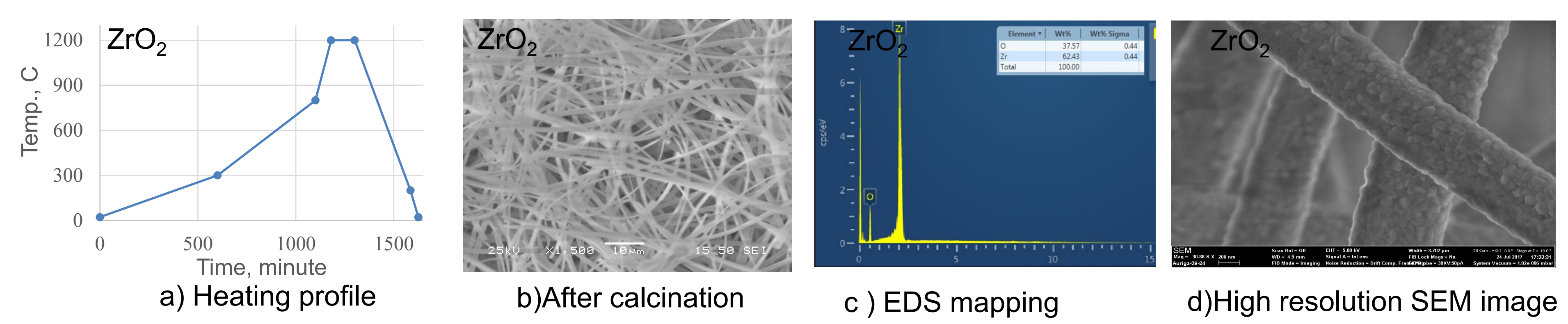

c) EDS mapping

\section{Electrospinning process}

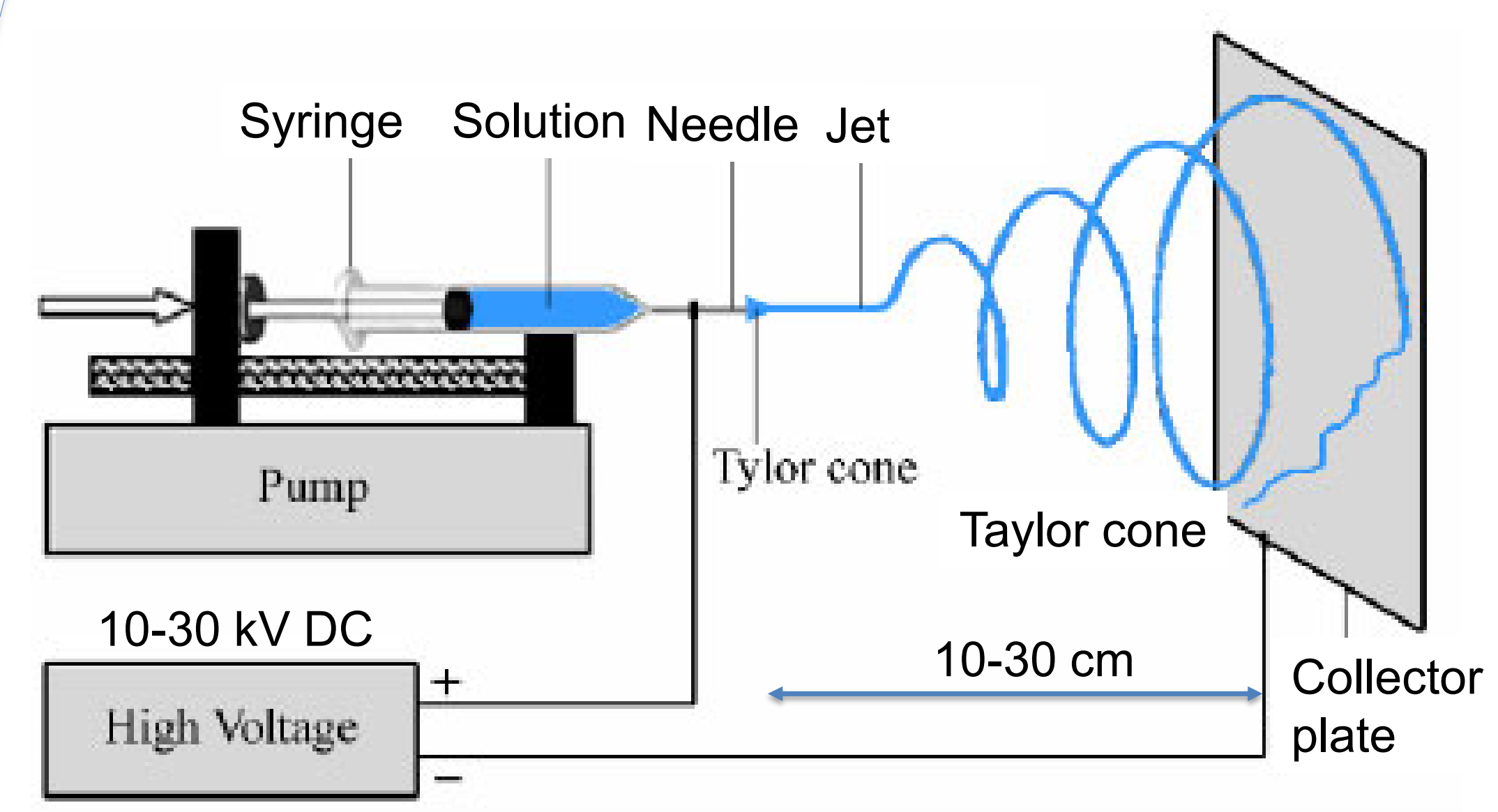

Process carried out at room temp. and atm. pressure

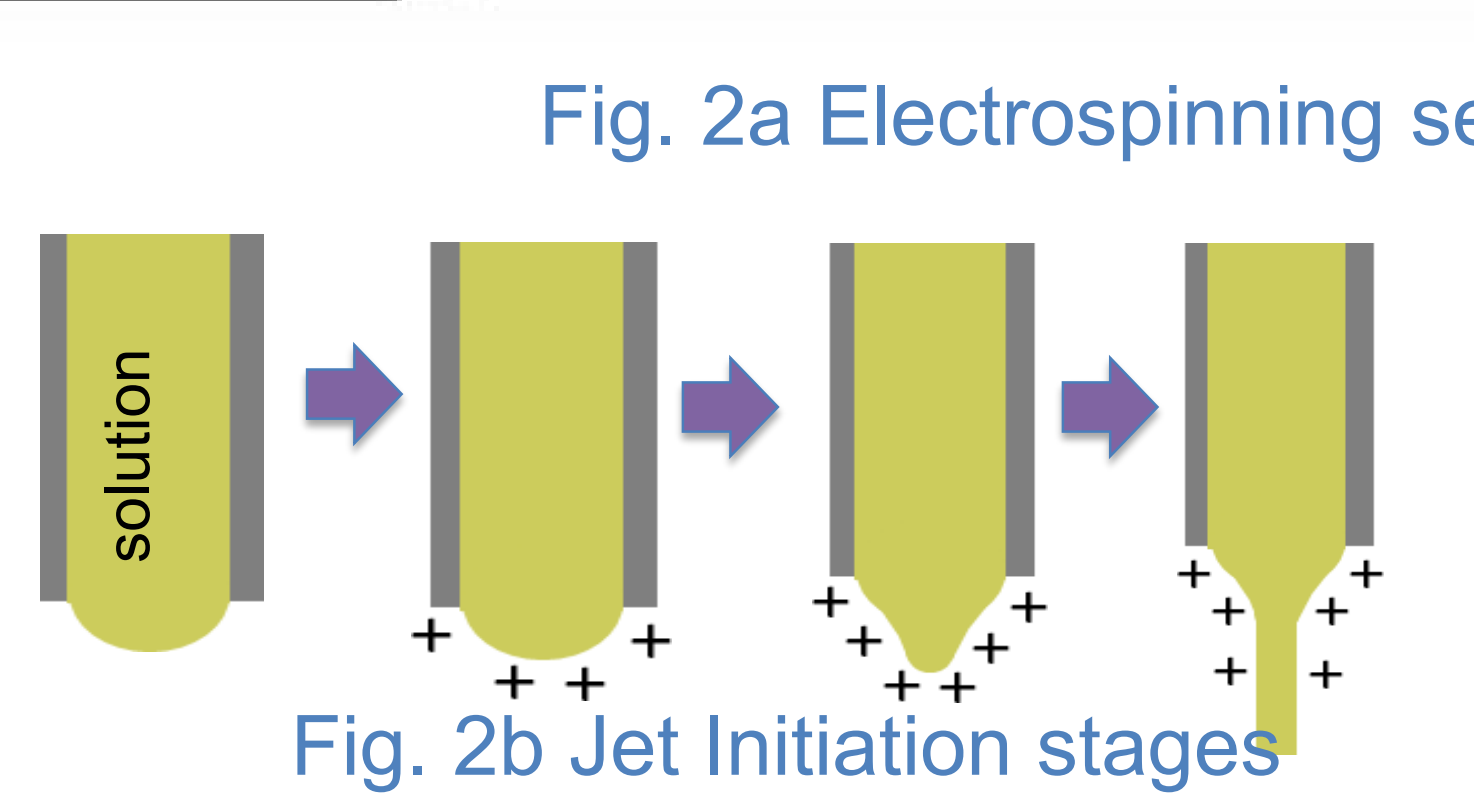

Process initiates when electrostatic repulsion over comes surface tension

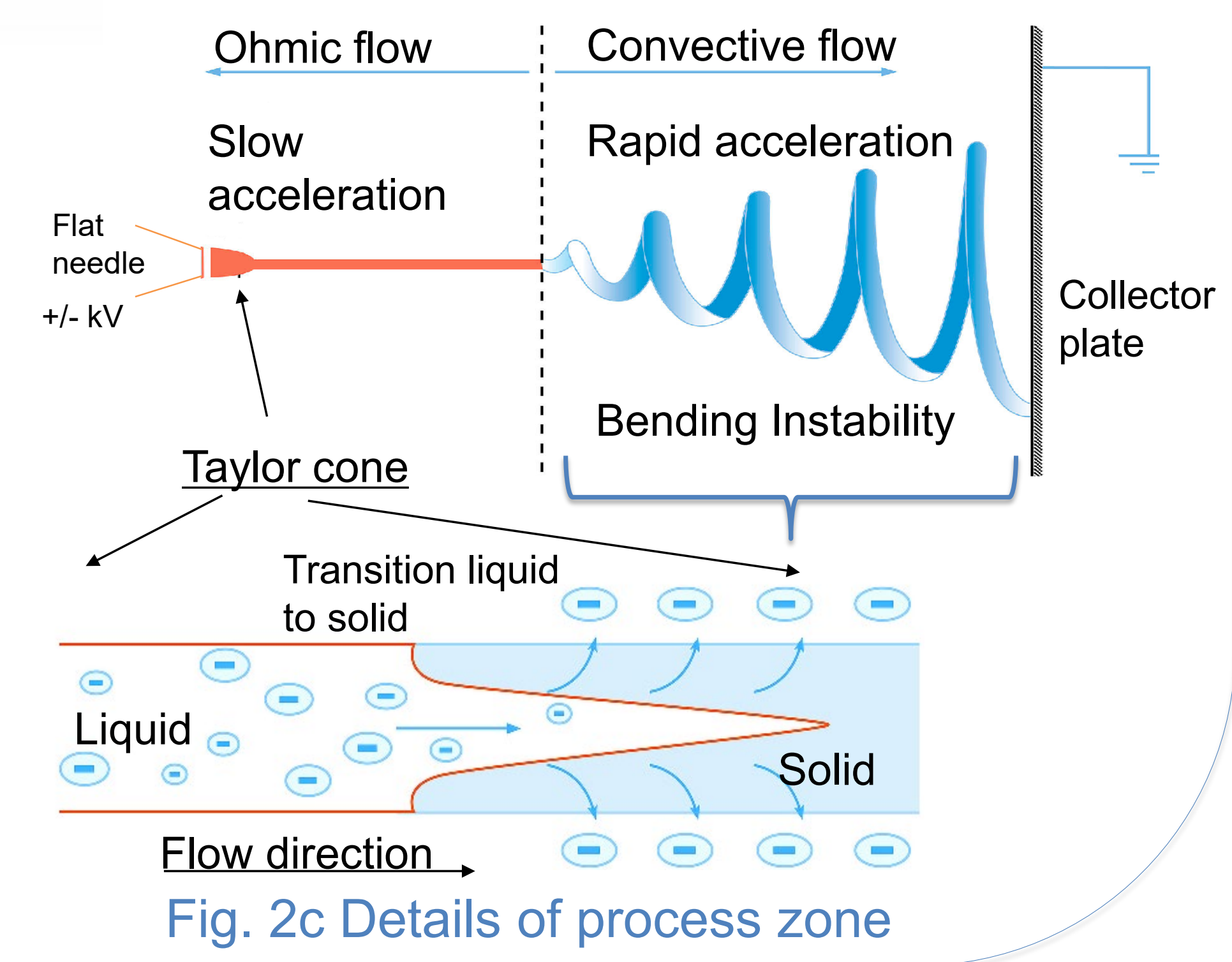

Thicker mat with ionizer

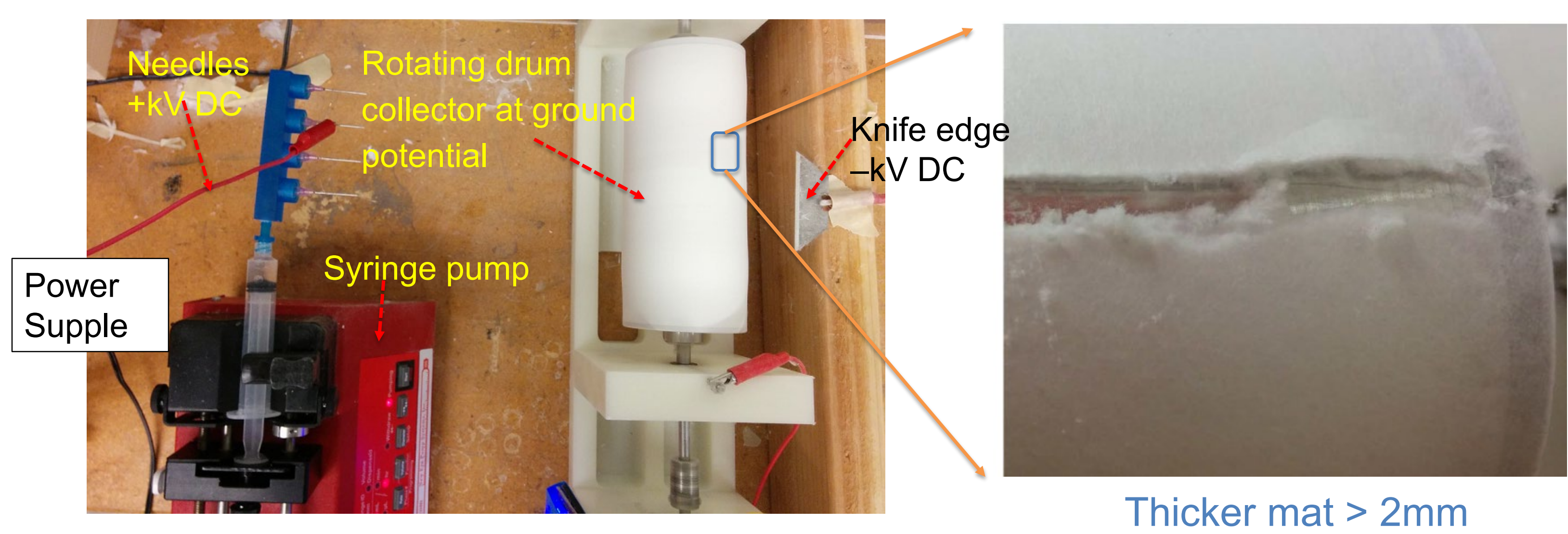

Fig. 4a Dual polarity electrospinning- ionizer

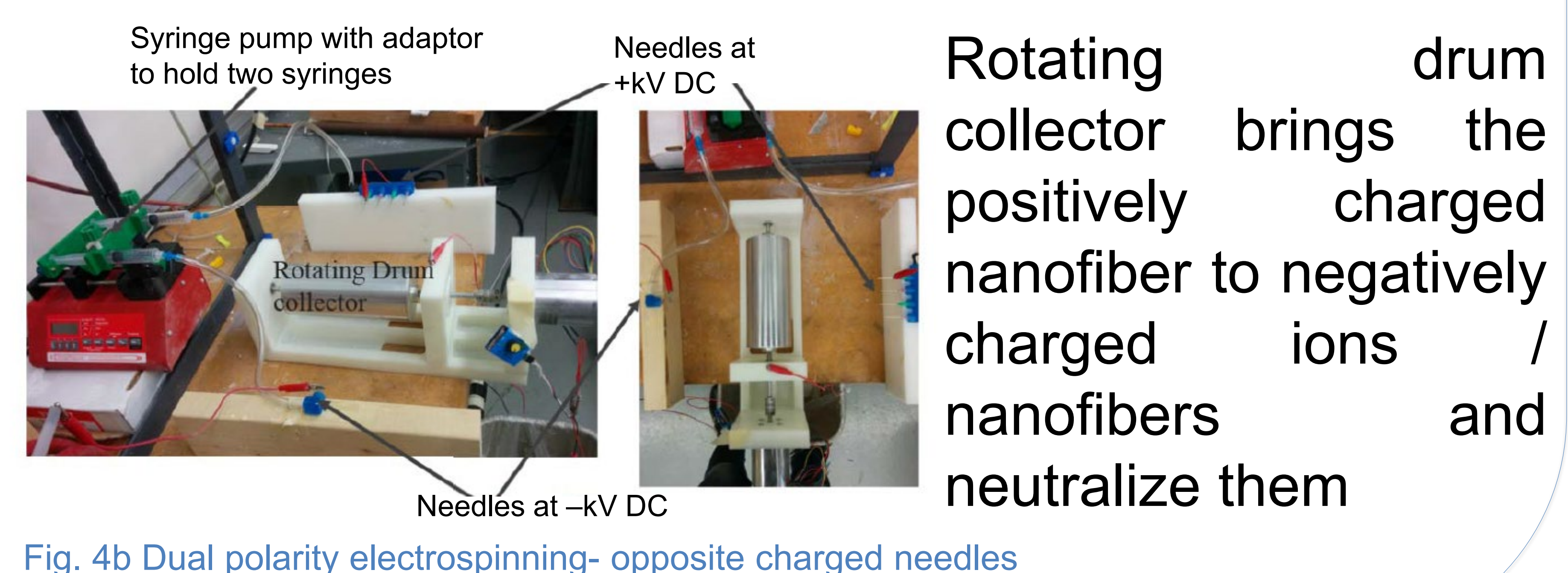

\section{Summary and Future work}

- Set up a low cost, low power, safer electrospinning unit.

- Success in fabricating metallic and ceramic nanofiber.

- Thicker nanofiber production using dual polarity spinning.

- Ceramic nanofiber looks promising as future candidate target material.

Future work

- Single fiber bending test for tensile strength.

- Single fiber thermal properties evaluation.

- Radiation damage studies using ion irradiation. 